BMC JOURNAL OF SCIENTIFIC RESEARCH
A Multidisciplinary Research Journal
ISSN: 2594-3421

\title{
Important ornamental plants of Bharatpur Metropolitan City, Nepal
}

\author{
Manoj K.L. Das* \\ manaojdas068@gmail.com
}

\begin{abstract}
The practices of decorating home gardens and public places by ornamental plants have been known from ancient time. The present study was destined to document about the circumstances of ornamental plants in Bharatpur Metropolitan City. Informations were collected by questionnaires, interview and direct observation. 90 ornamental plants were documented belonging to 49 families. Out of them, 42 plants belong to the herbs, 28 plants are shrubs, 14 plants are trees and 3 plants of each were represented as climbers and epiphytic herbs respectively. 59 plants were cultivated for flowers. The most dominant ornamental plants are Tagetes patula (saypatri) Rosa indica (rose), Hibiscus rosa sinensis (Ghanti phool) Bougainvellia glabra (Kagaje phool) and Aloe vera (Gheu Kumari). The Plate families like Malvaceae, Asteraceae, Apocyanaceae, Fabaceae and Euphorbiaceae are frequently found in study area.
\end{abstract}

Keyword: Ornamental plant, herb, shrub, trees, Bharatpur Metropolitan City

\section{Introduction}

The ornamental or garden plants are grown for the display of aesthetic features including flowers, leaves, scent, foliage texture, fruit, stem and bark. (Christenusz 2016). In some cases, unusual features may be considered to be of interest such as the thorns or spines of cacti. Flowers are immemorial source a source of inspiration of poet (Pandey 2005). The ornamental plants are cultivated for decorative purpose landscape, design projects, as house plants, cut flowers, religious ceremony and wedding. The cultivation of these plants are called as floriculture a major branch of horticulture (While field 2002). The love of flowers goes back to thousands of year in history of mankind. Egyptians and Assyrain had launched the art of cultivation of ornamental plants (Bose, 1989).

The Bharatpur Metropolitan City is the main business and medical hub of Nepal and head quarter of Chitwan Province number 3. Chitwan has a tropical flora and great biodivertifaction. The first national park, Chitwan National Park of Nepal is situated as neighbor of Bharatpur MC. The Chitwan is located in the tropical zone of inner Terai region of Nepal. Geographically; it is situated in nearly central part of Nepal lies between Chure and Mahabharat range and about 415-650 mt. in altitude. The Chitwan is located between latitude of $83^{\circ} 55^{\prime} \mathrm{w}$ to $84^{\circ} 48^{\prime} \mathrm{w}$ and longitude $27^{\circ} 21^{\prime} \mathrm{N}$ to $27^{\circ} 46^{\prime} \mathrm{N}$. The area of Chitwan is about 2221 square Kilometer. This district has a lot of tropical flora. The vegetation of Chitwan is described in relation to its bioclimatic zones, altitude, latitude and pattern of rain fall (Das, 2016).

\footnotetext{
* Dr. Das is Reader in Botany at Birendra Multiple Campus
} 
Most of the people of Chitwan are plants lovers because the ancestries of them are basically farmers in prior. There are about a dozen of nurseries are established in the Bharatpur. The Aadhar Nursery is the most dominant. There are more than two hundred varieties of ornament is for like hedge, shrubs, palms, cacti, succulent plants, ornamentals, pteridophytes ornamental trees, climbers indoors plants, bulbous plants, lawn grasses, etc, are available (Koirala and Das 2017).

Ornamental plants of nurseries play important role in socio-economic impact in Bharatpur M.C. (Koirala and Das 2017). Beside these, the ornamental plants have high aesthetic value and plays significant role in controlling pollution and beauty of city. (Ripmeestre M. 2009) The present paper deals with the habit, plants to be used as ornamental purpose and life span of important ornamental plants of Bharatpur MC.

\section{Material and methods:}

The current research work was based on both primary and secondary data. The primary data were collected from the field visit, interview, direct observation informal discussion and secondary data were collected from the prevalent literature, textbooks, journals, research paper and different websites. For this research work, the researcher concerned with different nurseries, hospitals, hotels, collages and garden loving householder, from December to May, 2018 but this work was mainly focused on nurseries in Bharatpur MC.

\section{Result and discussion:}

The study area has a lot of ornamental plants diversity. These plants play remarkable role in many ways as a whole, 90 ornamental plants belonging to 49 families has been recorded in Bharatpur MC a 50 list of ornamental plants along with their scientific name, common name, family, habit, ornamental parts categories and growing pattern are mentioned in Appendix I.

Among the documented ornamental plans, the families Fabaceae and Asteraceae are dominant each family has eight genera. $2^{\text {nd }}$ dominant families are Apocyanaceae having five genera. The moderate families are Euphorbiaceae, Oleaceae, Commelinaceae, Orchidaseae, Aspergaceae. The less dominant families are as Phodelaceae, Arucuxiaceae, Nyctaginaceae, Cyperaceae, Ranunculaceae, Moraceae, Balsminaceae, Rubiaceae, Brassicaceae, Amaranthaceae, Convovulaceae, Cupressaceae, Acanthaceae, Lythaceae, Annoceae, Bignoniaceae, Violaceae, Portulaceae and Zamiaceae.

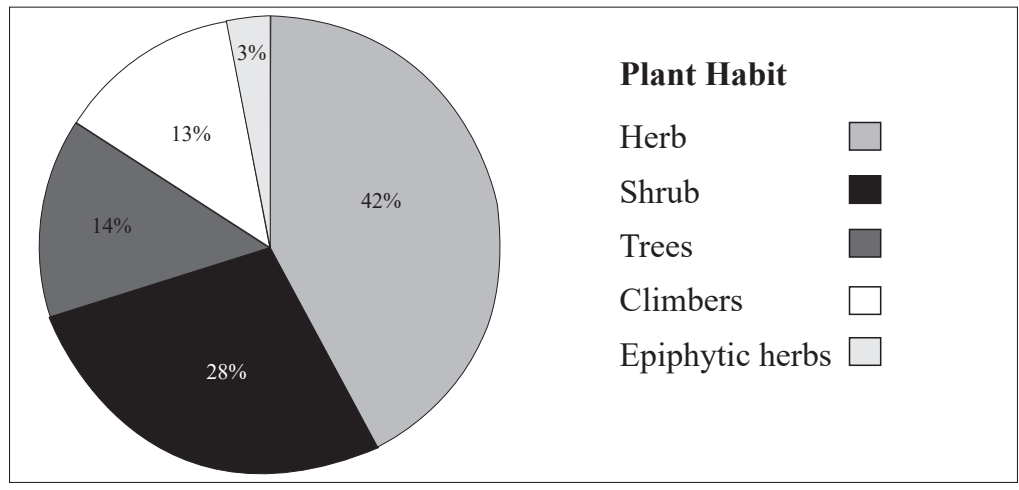


Out of ninety documented plants are harbs 28 plants are shrubs and 14 plants belong to trees whereas 3 plants are climbers and remaining 3 are epiphytec herbs.

Different ornamental plants are documented where flowers, leaves whole plant and fronds as the ornamental parts are identified. The predominant ornamented parts are flowers as it comprises 59 of them out of 90 documented plants 15 plants consists whole plant as ornamental value. Here, whole plants generally mean to palms, cacti and succulent. 10 plants have leaves for the ornamental purpose. 6 plants have leaves as ornamental purpose

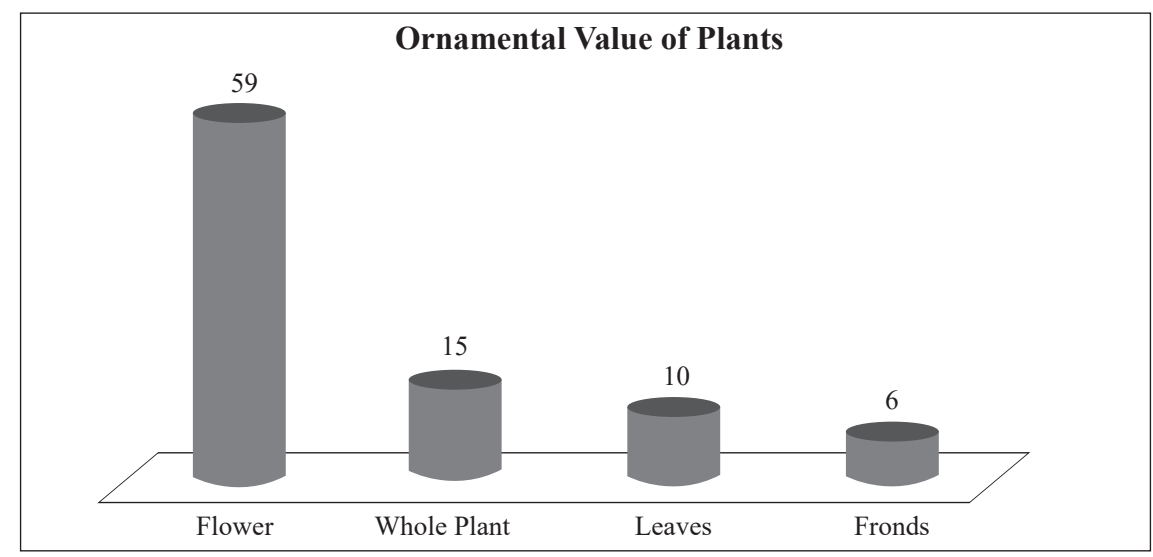

\section{Conclusion}

The current study contributes to the documentation important ornamental plants of Bharatpur MC. The Fabceae Asteraceae and Apocyanaceae families are important for the ornamental plants. The beautiful flowers of ornamental plants have great significant for attraction of human beings. Despite the development in the field floriculture, more than $50 \%$ of plants are still imported from India. The ornamental plants play significant role in decorating home garden, and public places. The concept of Bharatpur MC as "Green City" plays important role in reducing pollution increases the beauty of city and reduce the poverty in urban people.

\section{Reference}

Christennusz, M.T. and Byng J.W 2010; 'The number of known plants species in the world and its annual increase" phytotaxa, magnolia press 26(3): 20,2017

Whitefield P 2002; How to make a forest garden (pp113) Permanane publication ISBN 978-1-85629-008-7

Pandey DS and Singh NP 2005; Common flowers of India, Publication division, ministry of information and Broadcasting, Government of India

Bose TK and Yadav LP, Commercial flowers, Naya Prokash, Calculta

Koirala S and Das MKL 1917; 'Study of current status of ornamental plants and their documentation in Bharatpur Chitwa, dissertation, Birendra Multiple Campus.

Das M.K.L 2015; Enumeralition of wild flora of Birendra Multiple Campus Vivek Vo. 15 pp 183-191; ISSN: 23050-8639

Ripmeestra M, 2009; "Lawn" Encyclopedia of urban studies Ed. Ray Hutchinson 


\begin{tabular}{|c|c|c|c|c|c|c|c|c|c|c|c|c|c|c|c|c|c|c|}
\hline 象 & 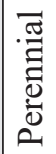 & 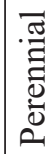 & 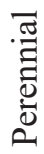 & $\begin{array}{l}\bar{\Xi} \\
\text { 主 }\end{array}$ & $\begin{array}{l}. \bar{\pi} \\
. \\
0 \\
0 \\
0 \\
0\end{array}$ & 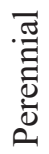 & 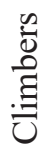 & 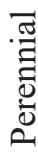 & 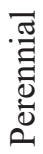 & 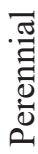 & $\begin{array}{l}\bar{\pi} \\
\text { J } \\
0 \\
0 \\
0 \\
0\end{array}$ & 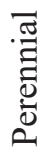 & 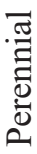 & 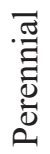 & 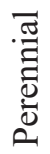 & 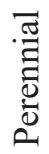 & 茎 & 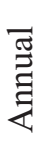 \\
\hline
\end{tabular}

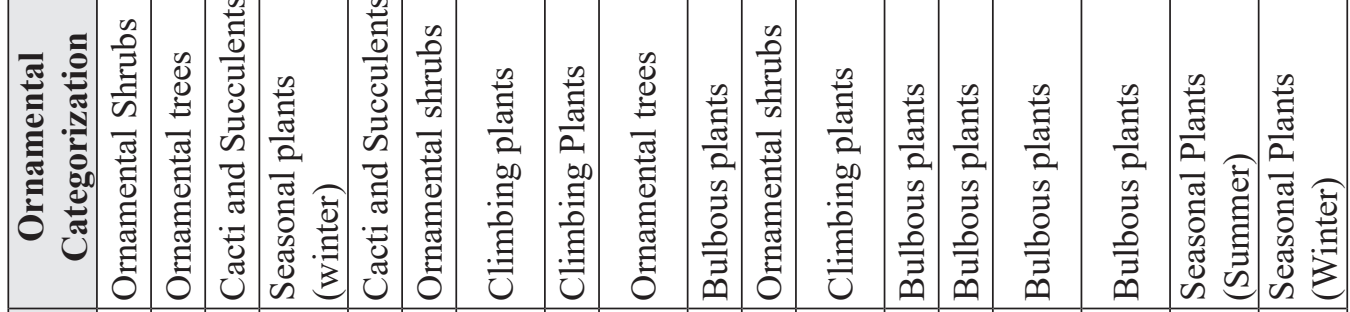

可

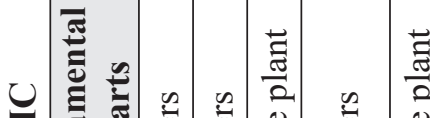

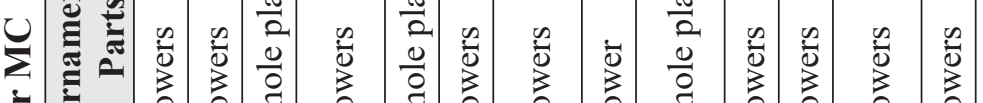

光

$\approx$ \&

章

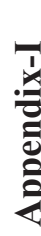

莺

¿

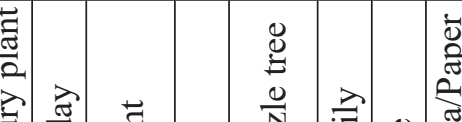

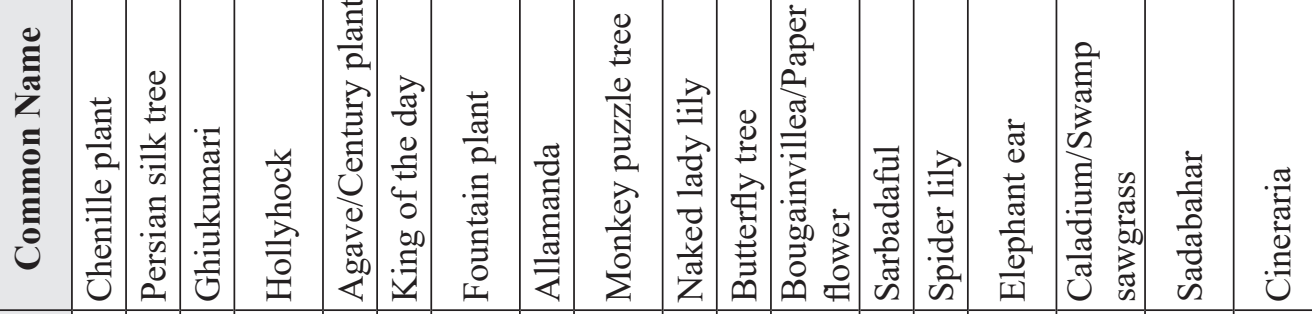

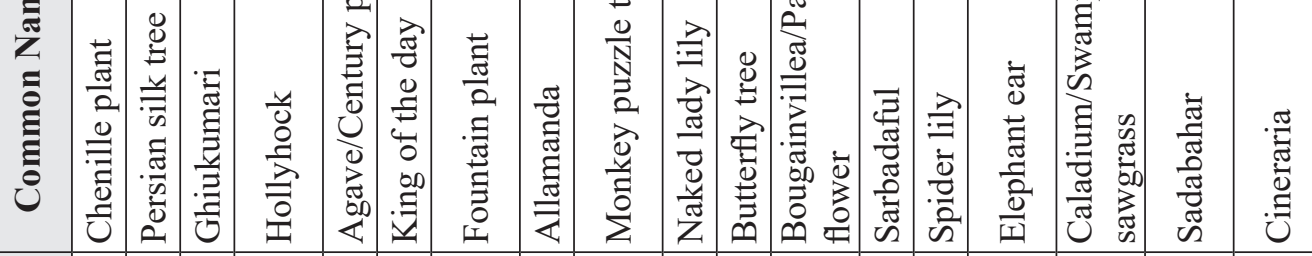

产

$\circ$ o

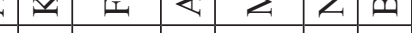

育

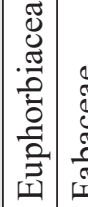

薃

总

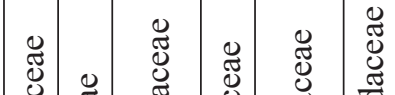

\&

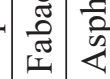

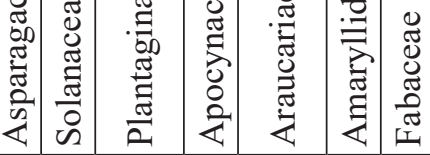

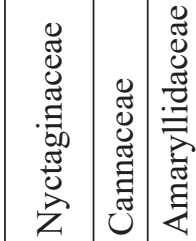

造

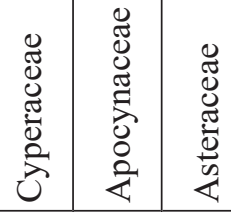

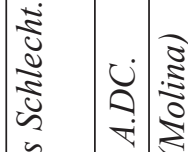

+ छें

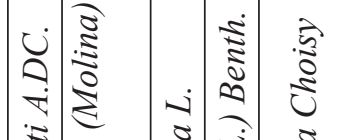




\begin{tabular}{|c|c|c|c|c|c|c|c|c|c|c|c|c|c|c|c|c|c|c|c|c|}
\hline 䲶 & 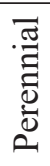 & 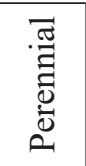 & 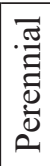 & 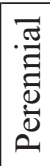 & 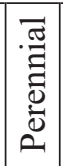 & $\begin{array}{l}\bar{\Xi} \\
\text { 芜 }\end{array}$ & $\begin{array}{l}\overline{\text { J }} \\
\text { 主 }\end{array}$ & $\begin{array}{l}\text { 吾 } \\
\text { 主 }\end{array}$ & 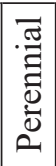 & 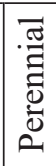 & 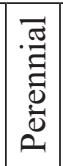 & 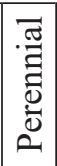 & 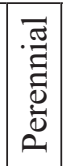 & 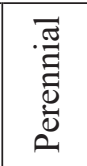 & 恶 & $\begin{array}{l}\text { 夏 } \\
\text { 妾 }\end{array}$ & $\begin{array}{l}\bar{\Xi} \\
\text { 芫 }\end{array}$ & 苞 & 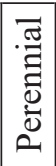 & $\begin{array}{l}\text { 夏 } \\
\text { 主 }\end{array}$ \\
\hline 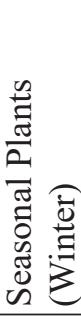 & 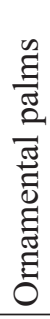 & 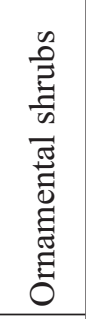 & 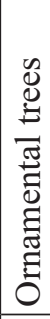 & 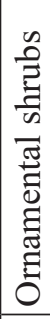 & 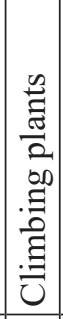 & 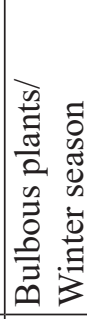 & 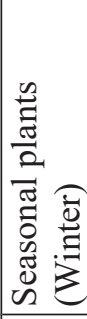 & 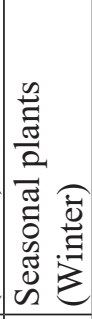 & 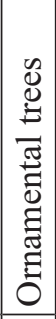 & 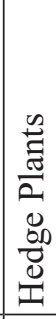 & 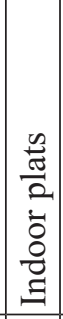 & 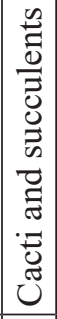 & 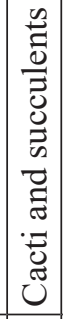 & 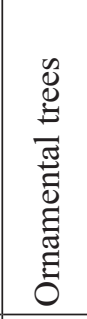 & $\begin{array}{l}\bar{T} \\
\tilde{0} \\
0 \\
0 \\
\tilde{D} \\
\tilde{D} \\
\end{array}$ & 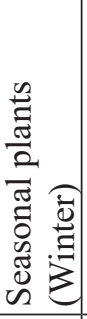 & 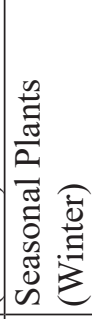 & 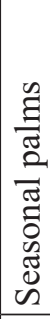 & 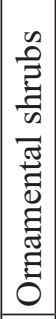 & 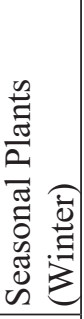 \\
\hline 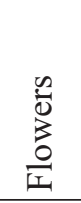 & $\begin{array}{l}\text { 䒕 } \\
\frac{0}{2} \\
\frac{0}{0} \\
0 \\
3\end{array}$ & 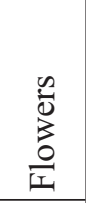 & $\begin{array}{l}0 \\
0 \\
0 \\
0 \\
0 \\
\frac{3}{I}\end{array}$ & $\begin{array}{l}0 \\
0 \\
0 \\
0 \\
0 \\
\end{array}$ & 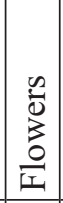 & $\begin{array}{l}\frac{\infty}{0} \\
\frac{0}{0} \\
\frac{0}{1}\end{array}$ & 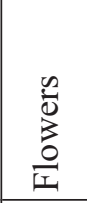 & $\begin{array}{l}\frac{\omega}{0} \\
\frac{3}{0} \\
\end{array}$ & \begin{tabular}{|l|}
0 \\
0 \\
0 \\
$\vdots$ \\
0 \\
$I$ \\
\end{tabular} & 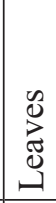 & 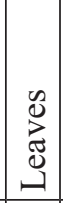 & 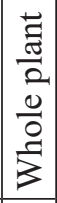 & 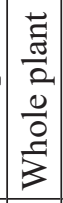 & $\begin{array}{l}\frac{\pi}{0} \\
\frac{0}{0} \\
\frac{3}{1}\end{array}$ & $\begin{array}{l}0 \\
0 \\
0 \\
\vdots \\
0 \\
0\end{array}$ & $\begin{array}{l}0 \\
0 \\
\frac{0}{0} \\
\frac{0}{1}\end{array}$ & 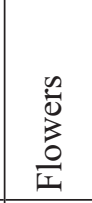 & $\begin{array}{l}0 \\
0 \\
0 \\
0 \\
0 \\
\\
\end{array}$ & \begin{tabular}{|l|}
0 \\
0 \\
0 \\
$\vdots$ \\
0 \\
$I$ \\
\end{tabular} & 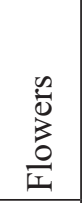 \\
\hline $\begin{array}{l}\stackrel{0}{0} \\
\stackrel{1}{I}\end{array}$ & 尝 & 昙 & 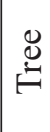 & $\frac{\hat{Z}}{\vec{Z}}$ & 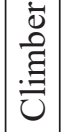 & $\begin{array}{l}\stackrel{0}{0} \\
\stackrel{1}{I}\end{array}$ & $\begin{array}{l}\stackrel{0}{0} \\
\frac{1}{1}\end{array}$ & $\begin{array}{l}\stackrel{0}{0} \\
\stackrel{1}{I}\end{array}$ & 总 & $\frac{\vec{Z}}{\vec{Z}}$ & 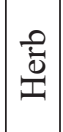 & 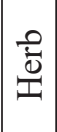 & $\begin{array}{l}\vec{Z} \\
\vec{D}\end{array}$ & ڤ્ટ & 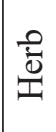 & $\begin{array}{l}\text { 离 } \\
\text { 至 }\end{array}$ & $\begin{array}{l}\text { 苛 } \\
\text { II }\end{array}$ & 这 & 昙 & $\begin{array}{l}\stackrel{0}{0} \\
\stackrel{0}{1}\end{array}$ \\
\hline 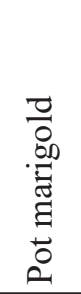 & 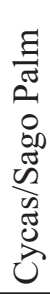 & 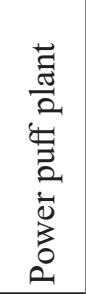 & 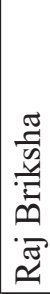 & $\begin{array}{l}\overline{0} \\
0 \\
0 \\
0 \\
20 \\
0 \\
0\end{array}$ & 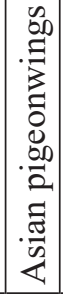 & 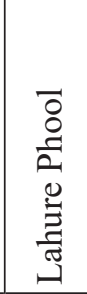 & 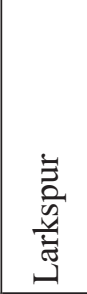 & 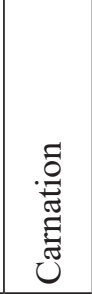 & 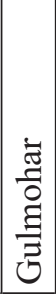 & $\begin{array}{l}0 \\
0 \\
0 \\
0 \\
0 \\
0 \\
0 \\
0 \\
0 \\
0 \\
0\end{array}$ & 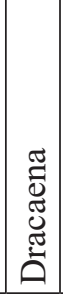 & 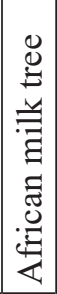 & 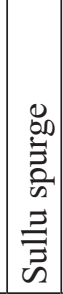 & 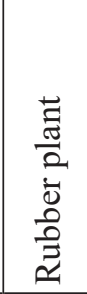 & 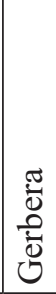 & 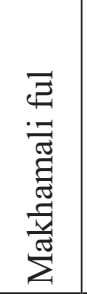 & 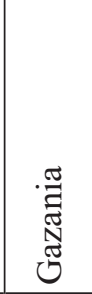 & 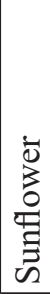 & 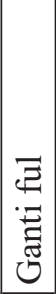 & 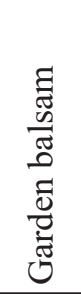 \\
\hline 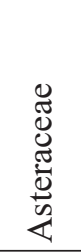 & 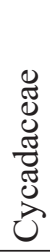 & 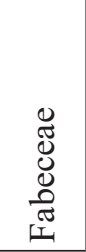 & 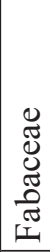 & 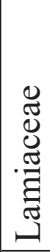 & 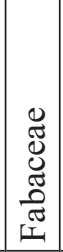 & 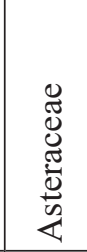 & 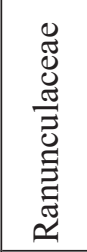 & 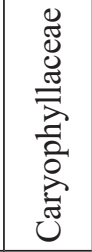 & 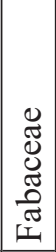 & 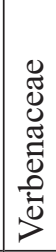 & 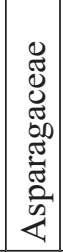 & 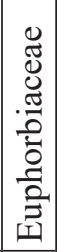 & 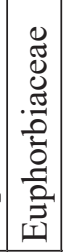 & 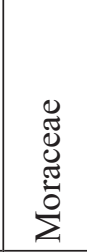 & 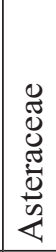 & 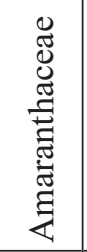 & 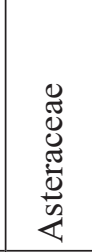 & 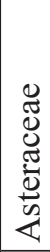 & 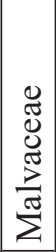 & 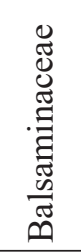 \\
\hline 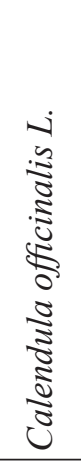 & 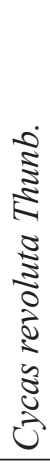 & 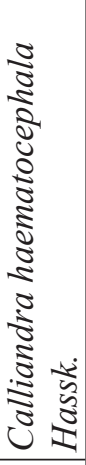 & 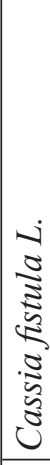 & $\begin{array}{c}5 \\
5 \\
\vdots \\
\vdots \\
\vdots \\
\vdots \\
0 \\
0 \\
0 \\
0 \\
0 \\
0\end{array}$ & 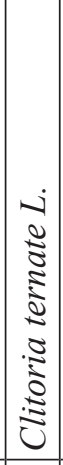 & 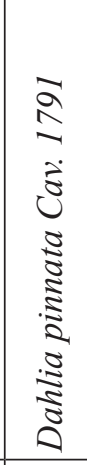 & 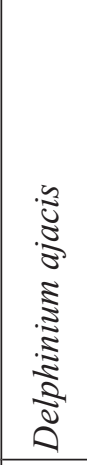 & 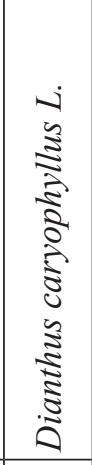 & 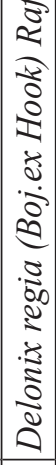 & \begin{tabular}{|c|}
0 \\
0 \\
0 \\
0 \\
0 \\
0 \\
0 \\
0 \\
0 \\
0 \\
0 \\
0 \\
0 \\
0
\end{tabular} & 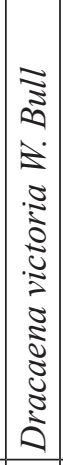 & 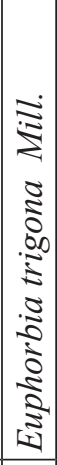 & 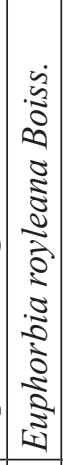 & 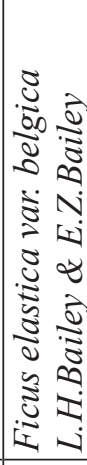 & $\begin{array}{c}2 \\
0 \\
0 \\
3 \\
0 \\
: 0 \\
0 \\
0 \\
0 \\
0\end{array}$ & $\begin{array}{c}1 \\
0 \\
5 \\
0 \\
0 \\
0 \\
0 \\
0 \\
5 \\
0 \\
5 \\
5 \\
5 \\
0 \\
0\end{array}$ & 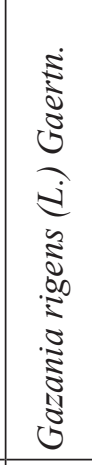 & 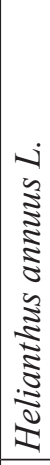 & 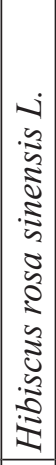 & 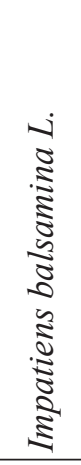 \\
\hline$\stackrel{a}{2}$ & $\widetilde{N}$ & $N$ & $\sim$ & N & $\sim$ & N & $\sim$ & $\sim$ & $N$ & N & $m$ & $m$ & $m$ & $m$ & $m$ & $m$ & $m$ & in & $m$ & $m$ \\
\hline
\end{tabular}




\begin{tabular}{|c|c|c|c|c|c|c|c|c|c|c|c|c|c|c|c|c|c|c|c|}
\hline $\begin{array}{l}\overline{\text { a }} \\
\text { 妾 }\end{array}$ & 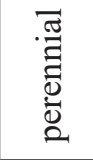 & 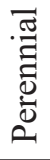 & 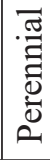 & 胥 & 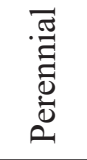 & 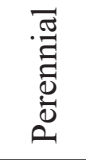 & 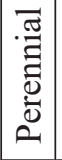 & 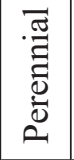 & 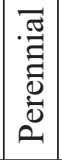 & 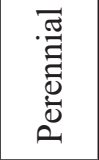 & 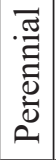 & 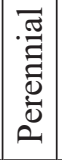 & 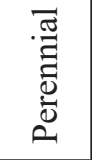 & $\begin{array}{l}\overline{\text { J }} \\
\text { 葛 }\end{array}$ & 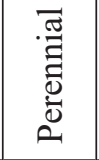 & 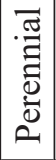 & 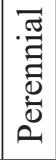 & 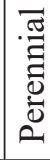 &. \\
\hline 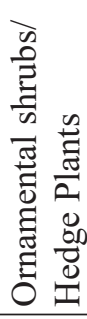 & 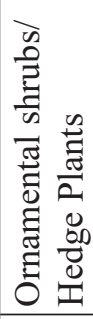 & 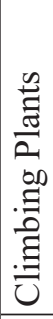 & 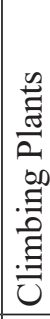 & 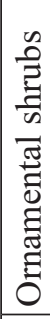 & 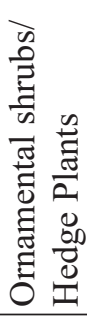 & 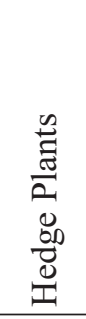 & 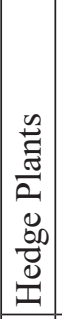 & 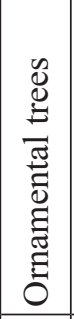 & 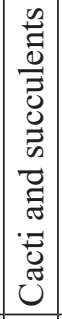 & 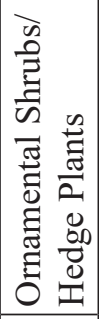 & 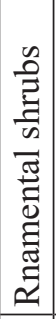 & 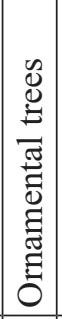 & 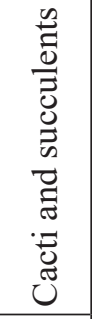 & 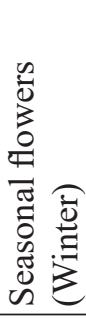 & 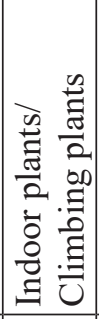 & 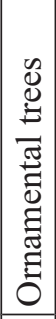 & 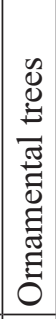 & 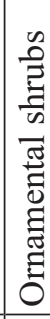 & 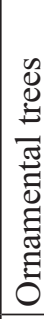 \\
\hline $\begin{array}{l}0 \\
0 \\
\frac{0}{2} \\
\frac{0}{1}\end{array}$ & 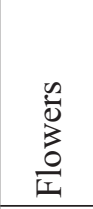 & 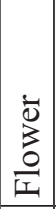 & $\begin{array}{l}0 \\
0 \\
0 \\
0 \\
0 \\
\end{array}$ & $\begin{array}{l}\text { 䓌 } \\
\frac{1}{2} \\
0 \\
0 \\
0 \\
\end{array}$ & 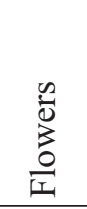 & 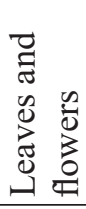 & \begin{tabular}{|c|}
$\frac{n}{0}$ \\
0 \\
0 \\
1 \\
\end{tabular} & $\begin{array}{l}0 \\
0 \\
0 \\
0 \\
0 \\
\end{array}$ & 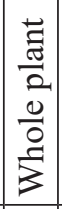 & $\begin{array}{l}\frac{\pi}{0} \\
\frac{0}{0} \\
\frac{3}{1}\end{array}$ & 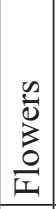 & $\left|\begin{array}{c}0 \\
0 \\
0 \\
0 \\
0 \\
\frac{0}{L}\end{array}\right|$ & $\begin{array}{l}\vec{\Xi} \\
\frac{\pi}{2} \\
\frac{0}{0} \\
0 \\
3\end{array}$ & 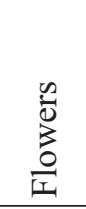 & 亗 & 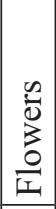 & $\mid \begin{array}{l}\overrightarrow{0} \\
\text { ज. } \\
0 \\
0 \\
0 \\
0 \\
3 \\
3\end{array}$ & $\begin{array}{c}0 \\
0 \\
\vdots \\
0 \\
\frac{0}{I I} \\
\end{array}$ & $\begin{array}{l}\frac{\pi}{2} \\
0 \\
\frac{1}{2} \\
\frac{1}{3}\end{array}$ \\
\hline $\begin{array}{l}\text { 苛 } \\
\text { II }\end{array}$ & $\frac{\hat{Z}}{\infty}$ & \begin{tabular}{|c|}
$\overline{0}$ \\
$\overline{0}$ \\
. \\
\end{tabular} & 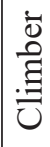 & 咅 & 昙 & 离 & $\frac{\vec{Z}}{\bar{\Xi}}$ & 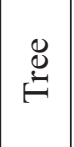 & $\begin{array}{l}0 \\
\stackrel{0}{0} \\
\end{array}$ & 竞 & 昙 & 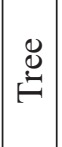 & $\begin{array}{l}\frac{0}{0} \\
\underline{I}\end{array}$ & 离 & $\begin{array}{l}\stackrel{0}{0} \\
\stackrel{1}{1}\end{array}$ & 导 & 导 & 兰 & \\
\hline 胥 & $\underset{\widetilde{\sigma}}{\tilde{x}}$ & 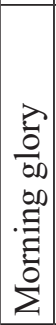 & 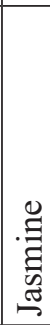 & 节 & 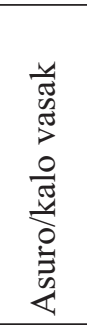 & $\begin{array}{l}\stackrel{\Xi}{\Xi} \\
\stackrel{\Xi}{ \pm}\end{array}$ & 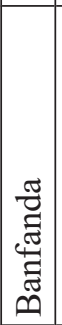 & 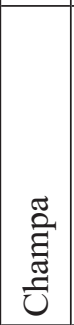 & $\stackrel{\mathscr{C}}{己}$ & 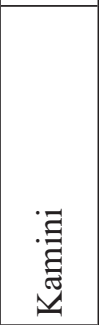 & 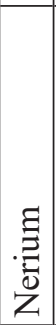 & 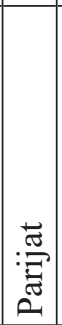 & 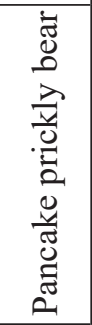 & $\frac{\hat{2}}{2}$ & 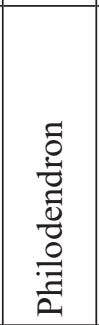 & 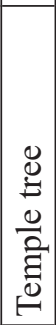 & $\begin{array}{l}\tilde{y} \\
\frac{\tilde{y}}{0} \\
\frac{5}{4} \\
\sum\end{array}$ & 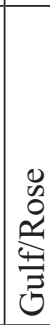 & 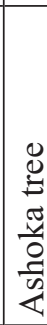 \\
\hline 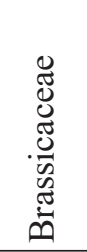 & 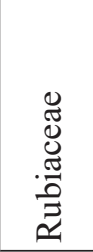 & \begin{tabular}{|l|} 
\\
$\tilde{d}$ \\
0 \\
0 \\
0 \\
3 \\
3 \\
0 \\
0 \\
0 \\
0 \\
\end{tabular} & $\begin{array}{l}\mathscr{E} \\
\mathbb{E} \\
\mathscr{E} \\
\mathbb{E} \\
0\end{array}$ & 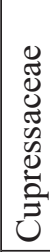 & 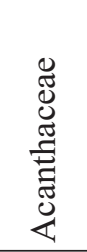 & 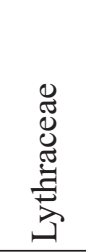 & 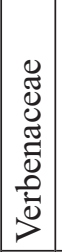 & 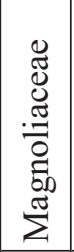 & 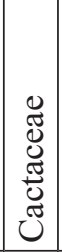 & $\begin{array}{l}\mathbb{\Xi} \\
\mathbb{\Xi} \\
\tilde{\Xi} \\
\Xi\end{array}$ & 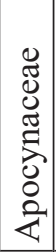 & 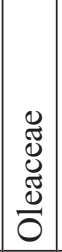 & 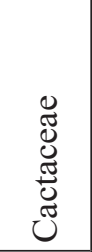 & 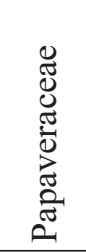 & 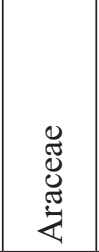 & 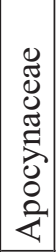 & 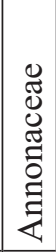 & 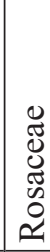 & $\begin{array}{l}\mathscr{\Xi} \\
\mathbb{\Xi}\end{array}$ \\
\hline 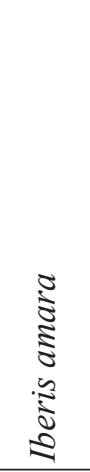 & 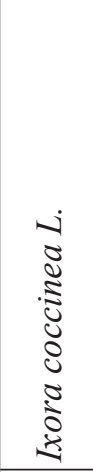 & 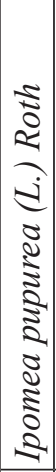 & 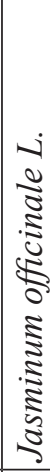 & 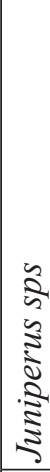 & 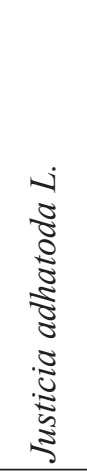 & 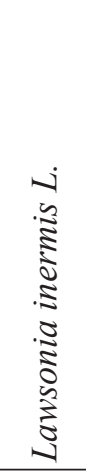 & 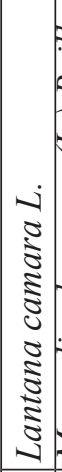 & 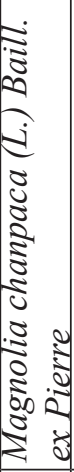 & 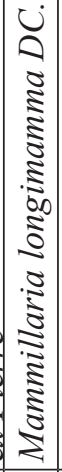 & 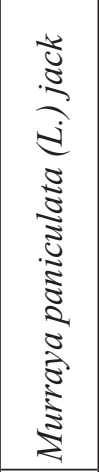 & 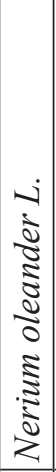 & 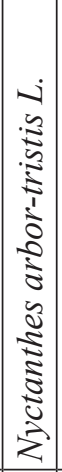 & 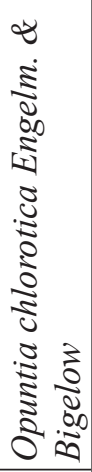 & 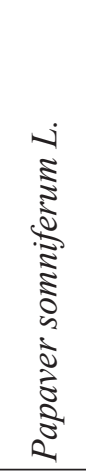 & $\begin{array}{l}5 \\
5 \\
5 \\
0 \\
0 \\
0 \\
0 \\
0 \\
\frac{1}{2} \\
\frac{1}{2}\end{array}$ & 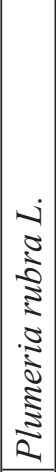 & 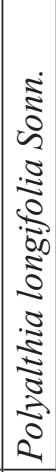 & $\begin{array}{l}\tilde{Z} \\
\varpi \\
2 \\
2\end{array}$ & $\begin{array}{l} \\
\tilde{y} \\
0 \\
0 \\
0 \\
0\end{array}$ \\
\hline 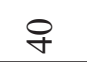 & $\checkmark$ & 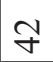 & 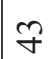 & f & $\forall$ & + & 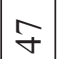 & チ & 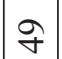 & $n$ & $n$ & $n$ & in & $n$ & in & $n$ & in & $\sim n$ & \\
\hline
\end{tabular}




\begin{tabular}{|c|c|c|c|c|c|c|c|c|c|c|c|c|c|c|c|c|}
\hline 丞 & 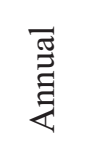 & 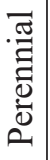 & 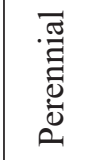 & $\begin{array}{l}\cdot \bar{J} \\
\overrightarrow{0} \\
0 \\
0 \\
0\end{array}$ & 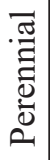 & 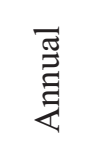 & 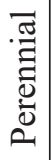 & $\mid \begin{array}{c}\cdot \vec{\pi} \\
\vec{J} \\
\overline{0} \\
0 \\
0 \\
0\end{array}$ & 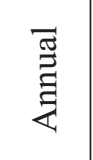 & 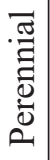 & 敖 & 敖 & 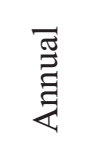 & 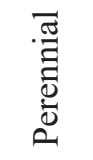 & 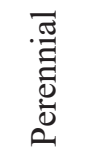 & 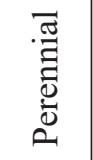 \\
\hline 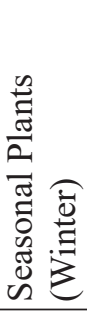 & 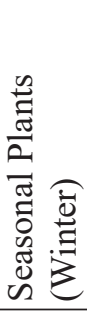 & 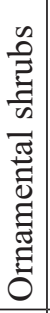 & 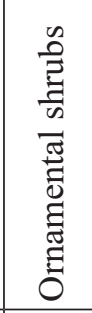 & 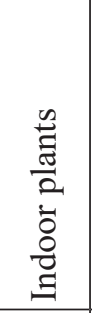 & 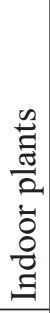 & 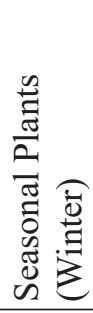 & 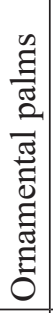 & 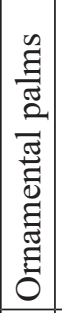 & 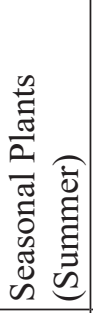 & 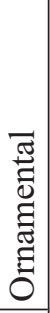 & 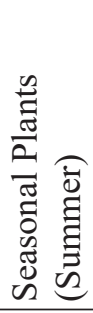 & 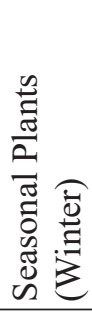 & 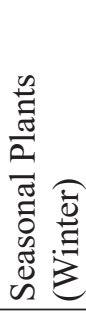 & 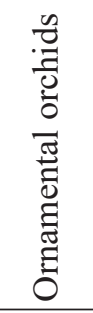 & 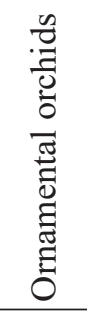 & 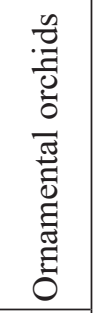 \\
\hline $\begin{array}{l}\frac{0}{0} \\
0 \\
0 \\
\frac{0}{1}\end{array}$ & $\begin{array}{l}n \\
\frac{0}{0} \\
\frac{0}{2} \\
\frac{0}{I}\end{array}$ & $\begin{array}{l}0 \\
0 \\
3 \\
0 \\
1 \\
\end{array}$ & $\begin{array}{l}\frac{0}{0} \\
0 \\
0 \\
0 \\
1\end{array}$ & 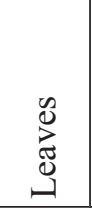 & 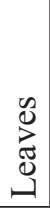 & $\begin{array}{l}0 \\
\frac{0}{0} \\
\frac{0}{2} \\
1\end{array}$ & 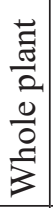 & $\left|\begin{array}{c}\overrightarrow{0} \\
\frac{\pi}{2} \\
0 \\
0 \\
0 \\
3 \\
3\end{array}\right|$ & $\begin{array}{l}0 \\
0 \\
\frac{0}{0} \\
\frac{0}{1}\end{array}$ & $\begin{array}{l}0 \\
0 \\
0 \\
0 \\
0 \\
1\end{array}$ & 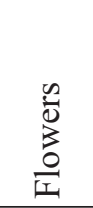 & 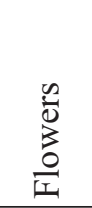 & $\begin{array}{l}n \\
\frac{0}{0} \\
\frac{3}{1} \\
\frac{0}{1}\end{array}$ & $\begin{array}{l}\frac{\pi}{0} \\
\frac{0}{1} \\
\end{array}$ & $\begin{array}{l}0 \\
0 \\
\frac{0}{0} \\
\frac{0}{1}\end{array}$ & $\begin{array}{l}0 \\
0 \\
0 \\
0 \\
0 \\
\frac{0}{1}\end{array}$ \\
\hline $\begin{array}{l}\stackrel{0}{0} \\
\stackrel{1}{I}\end{array}$ & $\begin{array}{l}\stackrel{0}{0} \\
\stackrel{0}{ \pm}\end{array}$ & 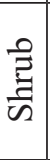 & 兰 & 离 & $\begin{array}{l}0 \\
\stackrel{0}{0} \\
\stackrel{1}{1}\end{array}$ & $\begin{array}{l}\stackrel{0}{0} \\
\stackrel{0}{I}\end{array}$ & 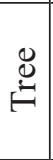 & 导 & 总 & $\frac{\hat{E}}{\vec{E}}$ & $\begin{array}{l}\stackrel{0}{0} \\
\stackrel{0}{I}\end{array}$ & $\begin{array}{l}\stackrel{0}{0} \\
\stackrel{0}{1}\end{array}$ & $\begin{array}{l}\stackrel{0}{0} \\
\stackrel{ \pm}{=}\end{array}$ & 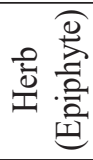 & 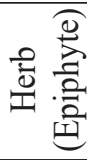 & 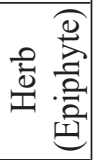 \\
\hline $\begin{array}{l}\mathscr{E}_{0} \\
\underset{\mathbb{D}}{\tilde{D}}\end{array}$ & 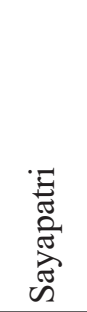 & 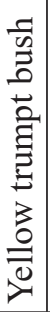 & 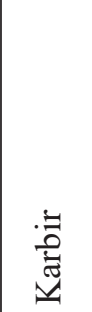 & $\begin{array}{l}\frac{\tilde{E}}{\tilde{E}} \\
\frac{\tilde{E}}{0} \\
\underline{\Xi}\end{array}$ & 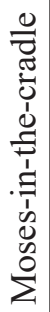 & 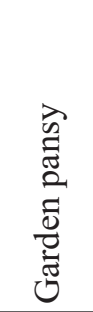 & 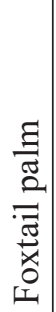 & 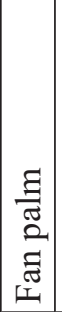 & 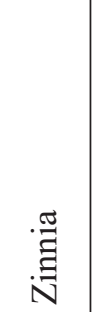 & 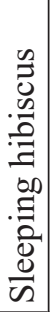 & $\begin{array}{l}0 \\
0 \\
0 \\
0 \\
0 \\
0 \\
0 \\
0 \\
\Sigma\end{array}$ & 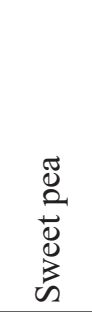 & $\frac{\ddot{o}}{\frac{a}{a}}$ & 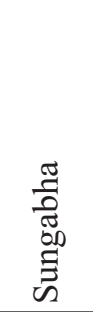 & 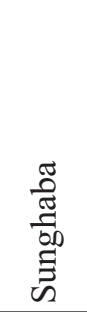 & 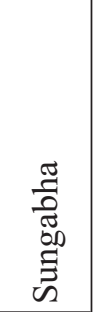 \\
\hline 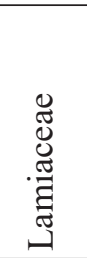 & 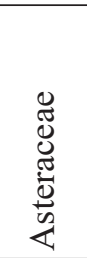 & 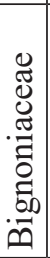 & 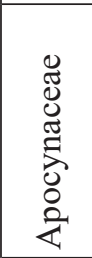 & 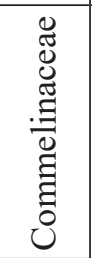 & 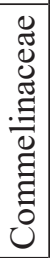 & 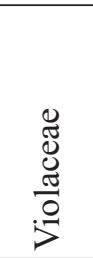 & 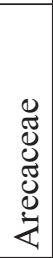 & 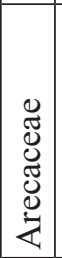 & 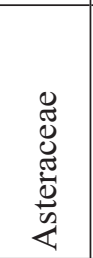 & 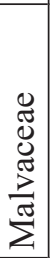 & 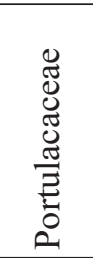 & 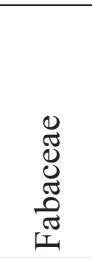 & 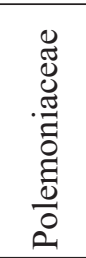 & 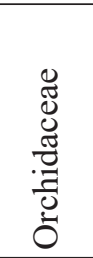 & 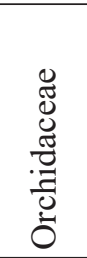 & 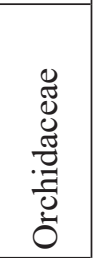 \\
\hline 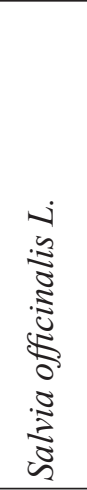 & $\begin{array}{l}\dot{3} \\
0 \\
0 \\
0 \\
0 \\
0 \\
3 \\
0 \\
0 \\
0 \\
0\end{array}$ & 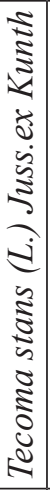 & 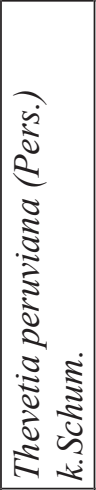 & 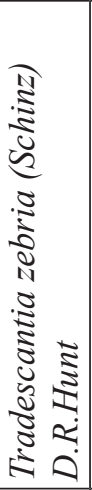 & 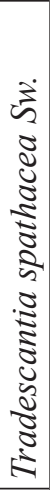 & $\begin{array}{l}0 \\
0 \\
0 \\
0 \\
\vdots \\
0 \\
0 \\
0 \\
0 \\
0 \\
0 \\
0 \\
0 \\
0 \\
0 \\
0 \\
0 \\
0 \\
0 \\
0 \\
0 \\
0\end{array}$ & 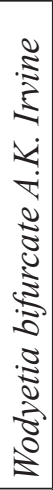 & 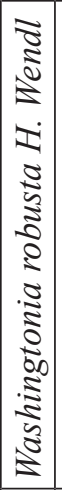 & 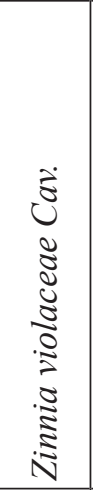 & 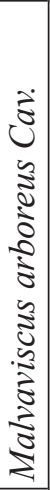 & \begin{tabular}{l}
0 \\
5 \\
0 \\
0 \\
0 \\
\multirow{3}{0}{} \\
0 \\
0 \\
0
\end{tabular} & 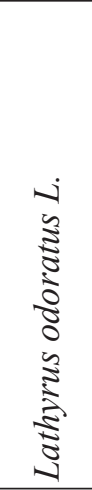 & $\begin{array}{l}\frac{2}{n} \\
\frac{5}{5} \\
\frac{a}{2}\end{array}$ & 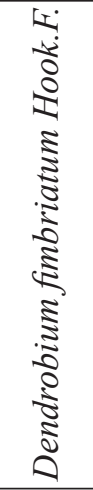 & 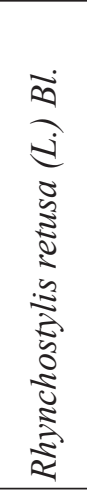 & 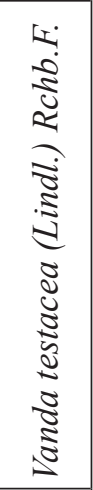 \\
\hline ஜ & 6 & ర్ & 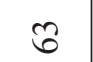 & tै & 0 & 8 & 6 & 0 & 6े & $\kappa$ & $\nabla$ & $\mathbb{N}$ & $\pi$ & $\underset{\sim}{ \pm}$ & $\stackrel{n}{n}$ & $\stackrel{2}{2}$ \\
\hline
\end{tabular}




\begin{tabular}{|c|c|c|c|c|c|c|c|c|c|c|c|c|c|}
\hline 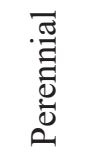 & 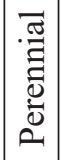 & 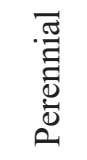 & 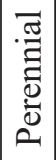 & 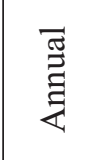 & 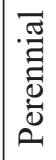 & 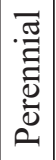 & 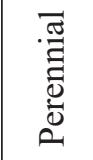 & 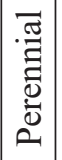 & 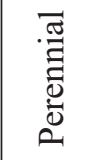 & 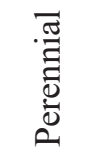 & 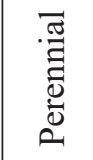 & $\begin{array}{c}. \bar{J} \\
\vec{U} \\
0 \\
0 \\
0 \\
0 \\
2\end{array}$ & 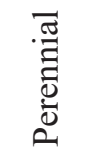 \\
\hline 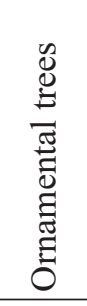 & 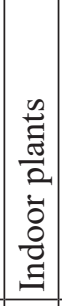 & 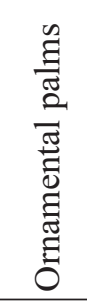 & 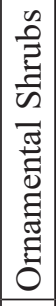 & 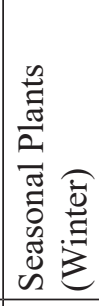 & 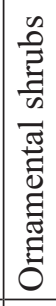 & 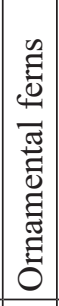 & 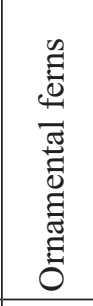 & 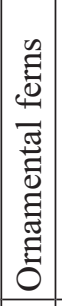 & 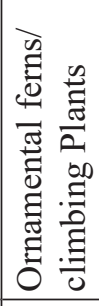 & 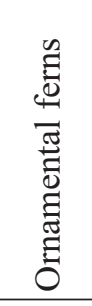 & 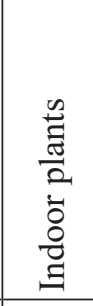 & 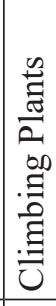 & 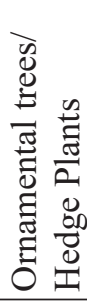 \\
\hline 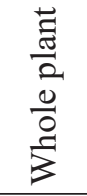 & 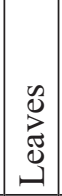 & 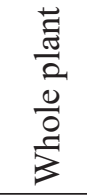 & 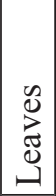 & $\begin{array}{l}\frac{2}{0} \\
\frac{0}{0} \\
\frac{0}{I}\end{array}$ & $\begin{array}{l}0 \\
0 \\
0 \\
0 \\
0 \\
1 \\
\end{array}$ & $\mid \begin{array}{c}\tilde{y} \\
0 \\
0 \\
0 \\
\dot{0}\end{array}$ & 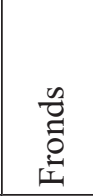 & $\mid \begin{array}{c}n \\
\tilde{0} \\
0 \\
0 \\
0\end{array}$ & 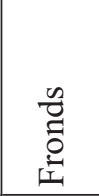 & 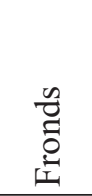 & 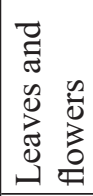 & $\begin{array}{l}0 \\
0 \\
0 \\
0 \\
0 \\
1 \\
\end{array}$ & 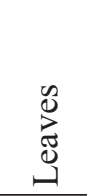 \\
\hline 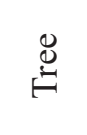 & 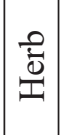 & 导 & 昙 & $\begin{array}{l}\stackrel{0}{0} \\
\stackrel{D}{I}\end{array}$ & $\frac{\vec{Z}}{\vec{D}}$ & $\begin{array}{l}\stackrel{0}{0} \\
\stackrel{ \pm}{ \pm}\end{array}$ & 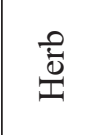 & $\mid \begin{array}{l}0 \\
\frac{0}{0} \\
0\end{array}$ & 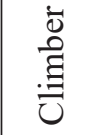 & $\begin{array}{l}\stackrel{0}{0} \\
\stackrel{0}{I}\end{array}$ & $\begin{array}{l}\stackrel{0}{0} \\
\stackrel{0}{I}\end{array}$ & $\begin{array}{l}\bar{\delta} \\
\text { है } \\
. \bar{U}\end{array}$ & 导 \\
\hline 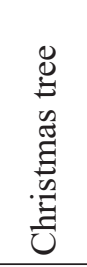 & 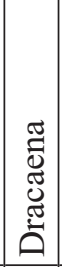 & 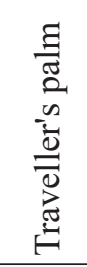 & 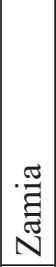 & $\begin{array}{l}\stackrel{\pi}{Z} \\
\vdots \\
0 \\
0\end{array}$ & 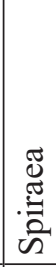 & 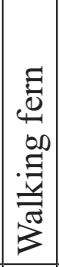 & & 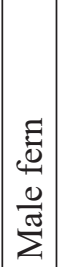 & 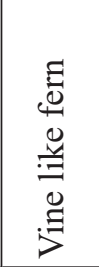 & 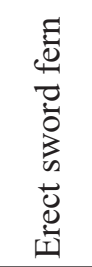 & 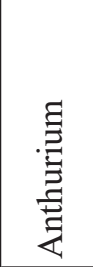 & 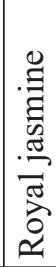 & $\frac{\stackrel{\sigma}{\Xi}}{\stackrel{\Xi}{E}}$ \\
\hline 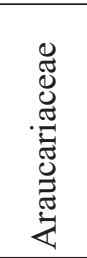 & 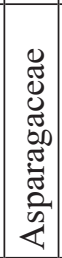 & 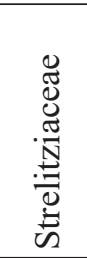 & 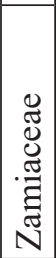 & 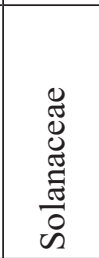 & 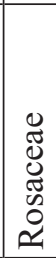 & 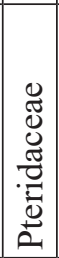 & 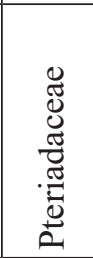 & 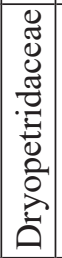 & 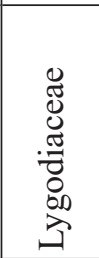 & 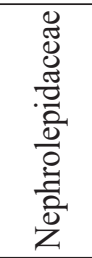 & 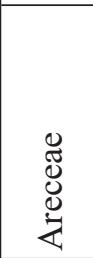 & 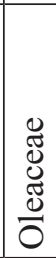 & 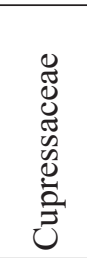 \\
\hline 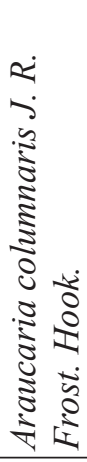 & 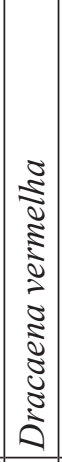 & 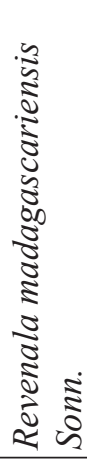 & 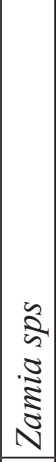 & 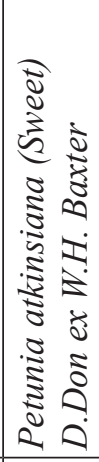 & 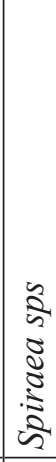 & 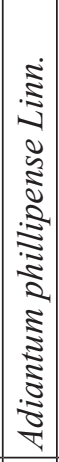 & 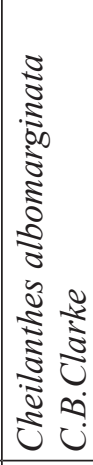 & $\mid \begin{array}{c}0 \\
0 \\
0 \\
0 \\
0 \\
0 \\
0 \\
0 \\
0 \\
0 \\
0 \\
0 \\
0 \\
0 \\
0 \\
0 \\
0 \\
0 \\
2 \\
0 \\
0 \\
0\end{array}$ & 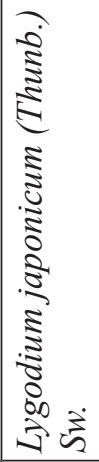 & 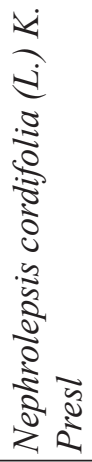 & 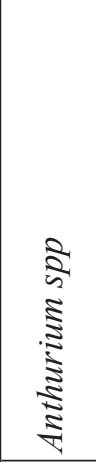 & 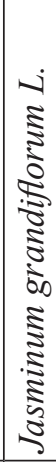 & 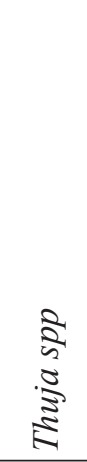 \\
\hline$\hat{\imath}$ & $\infty$ & 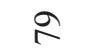 & $\infty$ & $\infty$ & $\widetilde{1}$ & $\infty$ & $\infty$ & $\infty$ & $\infty$ & $\hat{\infty}$ & $\infty$ & ஓ & ส \\
\hline
\end{tabular}

